Proyecciones Journal of Mathematics Vol. 36, N ${ }^{o}$ 1, pp. 81-94, March 2017. Universidad Católica del Norte Antofagasta - Chile

\title{
A weakened version of Davis-Choi-Jensen's inequality for normalised positive linear maps
}

\author{
S. S. Dragomir \\ Victoria University, Australia \\ Received : September 2016. Accepted : October 2016
}

\begin{abstract}
In this paper we show that the celebrated Davis-Choi-Jensen's inequality for normalised positive linear maps can be extended in a weakened form for convex functions. A reverse inequality and applications for important instances of convex (concave) functions are also given.
\end{abstract}

Subjclass : 47A63, 47A30, 15A60,.26D15, $26 D 10$.

Keywords : Operator convex functions, Convex functions, Power function, Logarithmic function, Exponential function. 


\section{Introduction}

The following result that provides an vector operator version for the Jensen inequality is well known, see for instance [6] or [7, p. 5]:

Theorem 1. Let $A$ be a selfadjoint operator on the Hilbert space $H$ and assume that $\operatorname{Sp}(A) \subseteq[m, M]$ for some scalars $m, M$ with $m<M$. If $f$ is a convex function on $[m, M]$, then

$$
f(\langle A x, x\rangle) \leq\langle f(A) x, x\rangle
$$

for each $x \in H$ with $\|x\|=1$.

As a special case of Theorem 1 we have the Hölder-McCarthy inequality [5]: Let $A$ be a selfadjoint positive operator on a Hilbert space $H$, then

(i) $\left\langle A^{r} x, x\right\rangle \geq\langle A x, x\rangle^{r}$ for all $r>1$ and $x \in H$ with $\|x\|=1$;

(ii) $\left\langle A^{r} x, x\right\rangle \leq\langle A x, x\rangle^{r}$ for all $0<r<1$ and $x \in H$ with $\|x\|=1$;

(iii) If $A$ is invertible, then $\left\langle A^{r} x, x\right\rangle \geq\langle A x, x\rangle^{r}$ for all $r<0$ and $x \in H$ with $\|x\|=1$.

In [2] (see also [3, p. 16]) we obtained the following additive reverse of (1.1):

Theorem 2. Let $I$ be an interval and $f: I \rightarrow \mathbf{R}$ be a convex and differ-

entiable function on $\stackrel{\circ}{I}$ (theinteriorof I) whosederivativef' is continuous on I. If $A$ is a selfadjoint operators on the Hilbert space $H$ with $\operatorname{Sp}(A) \subset I$, then

$$
\begin{gathered}
(0 \leq)\langle f(A) x, x\rangle-f(\langle A x, x\rangle) \\
\leq\left\langle f^{\prime}(A) A x, x\right\rangle-\langle A x, x\rangle \cdot\left\langle f^{\prime}(A) x, x\right\rangle
\end{gathered}
$$

for any $x \in H$ with $\|x\|=1$.

This is a generalization of the scalar discrete inequality obtained in [4]. For other reverse inequalities of this type see [3, p. 16].

The following particular cases are of interest: If $A$ is a selfadjoint operator on $H$, then we have the inequality:

$$
\begin{gathered}
(0 \leq)\langle\exp (A) x, x\rangle-\exp (\langle A x, x\rangle) \\
\leq\langle A \exp (A) x, x\rangle-\langle A x, x\rangle\langle\exp (A) x, x\rangle,
\end{gathered}
$$

for each $x \in H$ with $\|x\|=1$. 
Let $A$ be a positive definite operator on the Hilbert space $H$. Then we have the following inequality for the logarithm:

$$
\begin{aligned}
(0 & \leq) \ln (\langle A x, x\rangle)-\langle\ln (A) x, x\rangle \\
& \leq\langle A x, x\rangle\left\langle A^{-1} x, x\right\rangle-1,
\end{aligned}
$$

for each $x \in H$ with $\|x\|=1$.

If $p \geq 1$ and $A$ is a positive operator on $H$, then

$$
(0 \leq)\left\langle A^{p} x, x\right\rangle-\langle A x, x\rangle^{p} \leq p\left[\left\langle A^{p} x, x\right\rangle-\langle A x, x\rangle\left\langle A^{p-1} x, x\right\rangle\right]
$$

for each $x \in H$ with $\|x\|=1$. If $A$ is positive definite, then the inequality (1.5) also holds for $p<0$. If $0<p<1$ and $A$ is a positive definite operator then the reverse inequality also holds

$$
\left.(0 \leq)\langle A x, x\rangle^{p}-{ }^{p} x, x\right\rangle \leq p\left[\langle A x, x\rangle \cdot\left\langle A^{p-1} x, x\right\rangle-\left\langle A^{p} x, x\right\rangle\right]
$$

for each $x \in H$ with $\|x\|=1$.

Let $H$ be a complex Hilbert space and $\mathcal{B}(H)$, the Banach algebra of bounded linear operators acting on $H$. We denote by $\mathcal{B}_{h}(H)$ the semi-space of all selfadjoint operators in $\mathcal{B}(H)$. We denote by $\mathcal{B}^{+}(H)$ the convex cone of all positive operators on $H$ and by $\mathcal{B}^{++}(H)$ the convex cone of all positive definite operators on $H$.

Let $H, K$ be complex Hilbert spaces. Following [1] (see also [7, p. 18]) we can introduce the following definition:

Definition 1. A map $\Phi: \mathcal{B}(H) \rightarrow \mathcal{B}(K)$ is linear if it is additive and homogeneous, namely $\Phi(\lambda A+\mu B)=\lambda \Phi(A)+\mu \Phi(B)$

for any $\lambda, \mu \in \mathbf{C}$ and $A, B \in \mathcal{B}(H)$. The linear map $\Phi: \mathcal{B}(H) \rightarrow \mathcal{B}(K)$ is positive if it preserves the operator order,i.e. if $A \in \mathcal{B}^{+}(H)$ then $\Phi(A) \in$ $\mathcal{B}^{+}(K)$. We write $\Phi \in P[\mathcal{B}(H), \mathcal{B}(K)]$. The linear map $\Phi: \mathcal{B}(H) \rightarrow$ $\mathcal{B}(K)$ is normalised if it preserves the identity operator, i.e. $\Phi\left(1_{H}\right)=1_{K}$. We write $\Phi \in P_{N}[\mathcal{B}(H), \mathcal{B}(K)]$.

We observe that a positive linear map $\Phi$ preserves the order relation, namely

$$
A \leq B \text { implies } \Phi(A) \leq \Phi(B)
$$


and preserves the adjoint operation $\Phi\left(A^{*}\right)=\Phi(A)^{*}$. If $\Phi \in P_{N}[\mathcal{B}(H), \mathcal{B}(K)]$ and $\alpha 1_{H} \leq A \leq \beta 1_{H}$, then $\alpha 1_{K} \leq \Phi(A) \leq \beta 1_{K}$.

If the map $\Psi: \mathcal{B}(H) \rightarrow \mathcal{B}(K)$ is linear, positive and $\Psi\left(1_{H}\right) \in \mathcal{B}^{++}(K)$ then by putting $\Phi=\Psi^{-1 / 2}\left(1_{H}\right) \Psi \Psi^{-1 / 2}\left(1_{H}\right)$ we get that $\Phi \in P_{N}[\mathcal{B}(H), \mathcal{B}(K)]$, namely it is also normalised.

A real valued continuous function $f$ on an interval $I$ is said to be operator convex (concave) on $I$ if

$$
f((1-\lambda) A+\lambda B) \leq(\geq)(1-\lambda) f(A)+\lambda f(B)
$$

for all $\lambda \in[0,1]$ and for every selfadjoint operators $A, B \in \mathcal{B}(H)$ whose spectra are contained in $I$.

The following Jensen's type result is well known:

Theorem 3 (Davis-Choi-Jensen's Inequality). Let $f: I \rightarrow \mathbf{R}$ be an operator convex function on the interval $I$ and $\Phi \in P_{N}[\mathcal{B}(H), \mathcal{B}(K)]$, then for any selfadjoint operator $A$ whose spectrum is contained in $I$ we have

$$
f(\Phi(A)) \leq \Phi(f(A)) .
$$

We observe that if $\Psi \in P[\mathcal{B}(H), \mathcal{B}(K)]$ with $\Psi\left(1_{H}\right) \in \mathcal{B}^{++}(K)$, then by taking $\Phi=\Psi^{-1 / 2}\left(1_{H}\right) \Psi \Psi^{-1 / 2}\left(1_{H}\right)$ in (1.7) we get

$$
f\left(\Psi^{-1 / 2}\left(1_{H}\right) \Psi(A) \Psi^{-1 / 2}\left(1_{H}\right)\right) \leq \Psi^{-1 / 2}\left(1_{H}\right) \Psi(f(A)) \Psi^{-1 / 2}\left(1_{H}\right) .
$$

If we multiply both sides of this inequality by $\Psi^{1 / 2}\left(1_{H}\right)$ we get the following Davis-Choi-Jensen's inequality for general positive linear maps

$$
\begin{gathered}
\Psi^{1 / 2}\left(1_{H}\right) f\left(\Psi^{-1 / 2}\left(1_{H}\right) \Psi(A) \Psi^{-1 / 2}\left(1_{H}\right)\right) \Psi^{1 / 2}\left(1_{H}\right) \\
\leq \Psi(f(A)) .
\end{gathered}
$$

It is obvious that, by (1.7) we have the vector inequality

$$
\langle f(\Phi(A)) y, y\rangle \leq\langle\Phi(f(A)) y, y\rangle
$$

for any $y \in K$. By (1.1) we also have

$$
f(\langle\Phi(A) y, y\rangle) \leq\langle f(\Phi(A)) y, y\rangle
$$


for any $y \in K,\|y\|=1$. Therefore, for an operator convex function on $I$ we have

$$
f(\langle\Phi(A) y, y\rangle) \leq\langle f(\Phi(A)) y, y\rangle \leq\langle\Phi(f(A)) y, y\rangle
$$

for any $y \in K,\|y\|=1$.

It is then natural to ask the following question:

Does the inequality between the first and last term in (1.11) remains valid in the more general case of convex functions defined on the interval I $?$

A positive answer to this question and some reverse inequalities are provided below. Some applications for important instances of convex (concave) functions are also given.

\section{A Jensen's Type Inequality}

Suppose that $I$ is an interval of real numbers with interior $I$ and $f: I \rightarrow \mathbf{R}$ is a convex function on $I$. Then $f$ is continuous on $I$ and has finite left and right derivatives at each point of $I$. Moreover, if $t, s \in I$ and $t<s$, then $f_{-}^{\prime}(t) \leq f_{+}^{\prime}(t) \leq f_{-}^{\prime}(s) \leq f_{+}^{\prime}(s)$ which shows that both $f_{-}^{\prime}$ and $f_{+}^{\prime}$ are nondecreasing function on $I$. It is also known that a convex function must be differentiable except for at most countably many points.

For a convex function $f: I \rightarrow \mathbf{R}$, the subdifferential of $f$ denoted by $\partial f$ is the set of all functions $\varphi: I \rightarrow[-\infty, \infty]$ such that $\varphi(I) \subset \mathbf{R}$ and

$$
f(t) \geq f(a)+(t-a) \varphi(a) \text { for any } t, a \in I .
$$

It is also well known that if $f$ is convex on $I$, then $\partial f$ is nonempty, $f_{-}^{\prime}$, $f_{+}^{\prime} \in \partial f$ and if $\varphi \in \partial f$, then

$$
f_{-}^{\prime}(t) \leq \varphi(t) \leq f_{+}^{\prime}(t) \text { for any } t \in I .
$$

In particular, $\varphi$ is a nondecreasing function. If $f$ is differentiable and convex on $I$, then $\partial f=\left\{f^{\prime}\right\}$.

We have:

Theorem 1. Let $f: I \rightarrow \mathbf{R}$ be a convex function on the interval $I$ and $\Phi$ : $\mathcal{B}(H) \rightarrow \mathcal{B}(K)$ a normalised positive linear map. Then for any selfadjoint operator $A$ whose spectrum $\operatorname{Sp}(A)$ is contained in $I$ we have

$$
f(\langle\Phi(A) y, y\rangle) \leq\langle\Phi(f(A)) y, y\rangle
$$

for any $y \in K,\|y\|=1$. 
Proof. Let $m, M$ with $m<M$ and such that $\operatorname{Sp}(A) \subseteq[m, M] \subset I$. Then $m 1_{H} \leq A \leq M 1_{H}$ and since $\Phi \in P_{N}[\mathcal{B}(H), \mathcal{B}(K)]$ we have that $m 1_{K} \leq \Phi(A) \leq M 1_{K}$ showing that $\langle\Phi(A) y, y\rangle \in[m, M]$ for any $y \in K$, $\|y\|=1$. that

By the gradient inequality (2.1) we have for $a=\langle\Phi(A) y, y\rangle \in[m, M]$

$$
f(t) \geq f(\langle\Phi(A) y, y\rangle)+(t-\langle\Phi(A) y, y\rangle) \varphi(\langle\Phi(A) y, y\rangle)
$$

for any $t \in I$.

Using the continuous functional calculus for the operator $A$ we have for a fixed $y \in K$ with $\|y\|=1$ that

$$
f(A) \geq f(\langle\Phi(A) y, y\rangle) 1_{H}+\varphi(\langle\Phi(A) y, y\rangle)\left(A-\langle\Phi(A) y, y\rangle 1_{H}\right) .
$$

Since $\Phi \in P_{N}[\mathcal{B}(H), \mathcal{B}(K)]$, then by taking the functional $\Phi$ in the inequality (2.3) we get

$\Phi(f(A)) \geq f(\langle\Phi(A) y, y\rangle) 1_{K}+\varphi(\langle\Phi(A) y, y\rangle)\left(\Phi(A)-\langle\Phi(A) y, y\rangle 1_{K}\right)$

for any $y \in K$ with $\|y\|=1$.

This inequality is of interest in itself.

Taking the inner product in (2.4) we have for any $y \in K$ with $\|y\|=1$ that

$$
\begin{aligned}
& \langle\Phi(f(A)) y, y\rangle \\
& \geq f(\langle\Phi(A) y, y\rangle)\|y\|^{2}+\varphi(\langle\Phi(A) y, y\rangle)\left(\langle\Phi(A) y, y\rangle-\langle\Phi(A) y, y\rangle\|y\|^{2}\right) \\
& =f(\langle\Phi(A) y, y\rangle)
\end{aligned}
$$

and the inequality (2.2) is proved.

Corollary 1. Let $f: I \rightarrow \mathbf{R}$ be a convex function on the interval $I$ and $\Psi \in P[\mathcal{B}(H), \mathcal{B}(K)]$ with $\Psi\left(1_{H}\right) \in \mathcal{B}^{++}(K)$. Then for any selfadjoint operator $A$ whose spectrum $\operatorname{Sp}(A)$ is contained in $I$ we have 


$$
f\left(\frac{\langle\Psi(A) v, v\rangle}{\left\langle\Psi\left(1_{H}\right) v, v\right\rangle}\right) \leq \frac{\langle\Psi(f(A)) v, v\rangle}{\left\langle\Psi\left(1_{H}\right) v, v\right\rangle}
$$

for any $v \in K$ with $v \neq 0$.

Proof. If we write the inequality (2.2) for $\Phi=\Psi^{-1 / 2}\left(1_{H}\right) \Psi \Psi^{-1 / 2}\left(1_{H}\right)$ we have

$$
\begin{aligned}
& f\left(\left\langle\Psi^{-1 / 2}\left(1_{H}\right) \Psi(A) \Psi^{-1 / 2}\left(1_{H}\right) y, y\right\rangle\right) \\
& \leq\left\langle\Psi^{-1 / 2}\left(1_{H}\right) \Psi(f(A)) \Psi^{-1 / 2}\left(1_{H}\right) y, y\right\rangle
\end{aligned}
$$

for any $y \in K,\|y\|=1$.

Now, let $v \in K$ with $v \neq 0$ and take $y=\frac{1}{\left\|\Psi^{1 / 2}\left(1_{H}\right) v\right\|} \Psi^{1 / 2}\left(1_{H}\right) v$ in $(2)$ to get

$$
\begin{aligned}
& f\left(\left\langle\Psi^{-1 / 2}\left(1_{H}\right) \Psi(A) \Psi^{-1 / 2}\left(1_{H}\right) \frac{\Psi^{1 / 2}\left(1_{H}\right) v}{\left\|\Psi^{1 / 2}\left(1_{H}\right) v\right\|}, \frac{\Psi^{1 / 2}\left(1_{H}\right) v}{\left\|\Psi^{1 / 2}\left(1_{H}\right) v\right\|}\right\rangle\right) \\
\leq & \left\langle\Psi^{-1 / 2}\left(1_{H}\right) \Psi(f(A)) \Psi^{-1 / 2}\left(1_{H}\right) \frac{\Psi^{1 / 2}\left(1_{H}\right) v}{\left\|\Psi^{1 / 2}\left(1_{H}\right) v\right\|}, \frac{\Psi^{1 / 2}\left(1_{H}\right) v}{\left\|\Psi^{1 / 2}\left(1_{H}\right) v\right\|}\right\rangle
\end{aligned}
$$

that is equivalent to

$$
f\left(\left\langle\frac{\Psi(A) v}{\left\|\Psi^{1 / 2}\left(1_{H}\right) v\right\|}, \frac{v}{\left\|\Psi^{1 / 2}\left(1_{H}\right) v\right\|}\right\rangle\right) \leq\left\langle\frac{\Psi(f(A)) v}{\left\|\Psi^{1 / 2}\left(1_{H}\right) v\right\|}, \frac{v}{\left\|\Psi^{1 / 2}\left(1_{H}\right) v\right\|}\right\rangle
$$

and since

$$
\left\|\Psi^{1 / 2}\left(1_{H}\right) v\right\|^{2}=\left\langle\Psi\left(1_{H}\right) v, v\right\rangle
$$

for $v \in K$ with $v \neq 0$, then we obtain the desired inequality (2.5).

By taking some example of fundamental convex (concave) functions, we can state the following results:

Let $\Phi: \mathcal{B}(H) \rightarrow \mathcal{B}(K)$ be a normalised positive linear map.

(i) If $A$ is a selfadjoint operator on $H$ and $r \geq 1$, then we have

$$
|\langle\Phi(A) y, y\rangle|^{r} \leq\left\langle\Phi\left(|A|^{r}\right) y, y\right\rangle
$$


and in particular

$$
|\langle\Phi(A) y, y\rangle| \leq\langle\Phi(|A|) y, y\rangle
$$

for all $y \in K,\|y\|=1$. We have the norm inequality

$$
\|\Phi(A)\|^{r} \leq\left\|\Phi\left(|A|^{r}\right)\right\| .
$$

(ii) If $A$ is a positive operator on a Hilbert space $H$, then for any $p \geq 1$ $(p \in(0,1))$ we have

$$
\langle\Phi(A) y, y\rangle^{p} \leq(\geq)\left\langle\Phi\left(A^{p}\right) y, y\right\rangle
$$

for all $y \in K,\|y\|=1$. We have the norm inequality

$$
\|\Phi(A)\|^{p} \leq(\geq)\left\|\Phi\left(A^{p}\right)\right\| .
$$

If $A$ is a positive definite operator on a Hilbert space $H$, then for any $p<0$ we have

$$
\langle\Phi(A) y, y\rangle^{p} \leq\left\langle\Phi\left(A^{p}\right) y, y\right\rangle
$$

for all $y \in K,\|y\|=1$.

(iii) If $A$ is a selfadjoint operator on $H$ then we have

$$
\exp (\langle\Phi(A) y, y\rangle) \leq\langle\Phi(\exp (A)) y, y\rangle
$$

for all $y \in K,\|y\|=1$. We have the norm inequality

$$
\exp (\|\Phi(A)\|) \leq\|\Phi(\exp (A))\| .
$$

Let $P_{j} \in \mathcal{B}(H), j=1, \ldots, k$ be contractions with

$$
\sum_{j=1}^{k} P_{j}^{*} P_{j}=1_{H}
$$

The map $\Phi: \mathcal{B}(H) \rightarrow \mathcal{B}(H)$ defined by $[7]$

$$
\Phi(A):=\sum_{j=1}^{k} P_{j}^{*} A P_{j}
$$

is a normalized positive linear map on $\mathcal{B}(H)$. Therefore, if $f: I \rightarrow \mathbf{R}$ be a convex function on the interval $I$ and $A$ is selfadjoint operator whose spectrum $\operatorname{Sp}(A)$ is contained in $I$, we have by $(2.2)$ that 


$$
f\left(\sum_{j=1}^{k}\left\langle P_{j}^{*} A P_{j} y, y\right\rangle\right) \leq\left\langle\sum_{j=1}^{k} P_{j}^{*} f(A) P_{j} y, y\right\rangle
$$

for all $y \in K,\|y\|=1$.

If we take $k=1$ and $P_{1}=1_{H}$ in (2.15), then we recapture Jensen's inequality (1.1).

We then have for any selfadjoint operator $A$ and $r \geq 1$ that

$$
\left|\sum_{j=1}^{k}\left\langle P_{j}^{*} A P_{j} y, y\right\rangle\right|^{r} \leq\left\langle\sum_{j=1}^{k} P_{j}^{*}|A|^{r} P_{j} y, y\right\rangle
$$

and

$$
\exp \left(\sum_{j=1}^{k}\left\langle P_{j}^{*} A P_{j} y, y\right\rangle\right) \leq\left\langle\sum_{j=1}^{k} P_{j}^{*}(\exp A) P_{j} y, y\right\rangle
$$

for all $y \in K,\|y\|=1$. In the case $r=1$ we have

$$
\left|\sum_{j=1}^{k}\left\langle P_{j}^{*} A P_{j} y, y\right\rangle\right| \leq\left\langle\sum_{j=1}^{k} P_{j}^{*}|A| P_{j} y, y\right\rangle .
$$

By taking the supremum over $y \in K,\|y\|=1$ we also have the norm inequalities

$$
\left\|\sum_{j=1}^{k} P_{j}^{*} A P_{j}\right\|^{r} \leq\left\|\sum_{j=1}^{k} P_{j}^{*}|A|^{r} P_{j}\right\|, r \geq 1
$$

and

$$
\exp \left(\left\|\sum_{j=1}^{k} P_{j}^{*} A P_{j}\right\|\right) \leq\left\|\sum_{j=1}^{k} P_{j}^{*}(\exp A) P_{j}\right\| .
$$

In the case $r=1$ we have

$$
\left\|\sum_{j=1}^{k} P_{j}^{*} A P_{j}\right\| \leq\left\|\sum_{j=1}^{k} P_{j}^{*}|A|^{r} P_{j}\right\| .
$$

If $A$ is a positive operator on a Hilbert space $H$, then for any $p \in$ $(-\infty, 0) \cup[1, \infty)(p \in(0,1))$ we have by $(2.15)$ for power function that 


$$
\left\langle\sum_{j=1}^{k} P_{j}^{*} A P_{j} y, y\right\rangle^{p} \leq(\geq)\left\langle\sum_{j=1}^{k} P_{j}^{*} A^{p} P_{j} y, y\right\rangle
$$

for all $y \in K,\|y\|=1$.

If we take $k=1$ and $P_{1}=1_{H}$ in (2.22), then we recapture HölderMcCarthy's inequality.

By taking the supremum over $y \in K,\|y\|=1$ we also have the norm inequality

$$
\left\|\sum_{j=1}^{k} P_{j}^{*} A P_{j}\right\|^{p} \leq(\geq)\left\|\sum_{j=1}^{k} P_{j}^{*} A^{p} P_{j}\right\|,
$$

where $p \geq 1(p \in(0,1))$.

\section{A Reverse Inequality}

We have:

Theorem 1. Let $I$ be an interval and $f: I \rightarrow \mathbf{R}$ be a convex and differentiable function on $I$ whose derivative $f^{\prime}$ is continuous on $I$. If $\Phi: \mathcal{B}(H) \rightarrow$ $\mathcal{B}(K)$ is a normalised positive linear map and $A$ is a selfadjoint operators on the Hilbert space $H$ with $\operatorname{Sp}(A) \subset I$, then

$$
\begin{aligned}
0 & \leq\langle\Phi(f(A)) y, y\rangle-f(\langle\Phi(A) y, y\rangle) \\
& \leq\left\langle\Phi\left(A f^{\prime}(A)\right) y, y\right\rangle-\langle\Phi(A) y, y\rangle\left\langle\Phi\left(f^{\prime}(A)\right) y, y\right\rangle
\end{aligned}
$$

for any $y \in K,\|y\|=1$.

Proof. From the gradient inequality (2.1) we have

$$
f(t) \geq f(s)+(t-s) f^{\prime}(s)
$$

for any $t, s \in I$.

Let $y \in K,\|y\|=1$. If we take in (3.2) $t=\langle\Phi(A) y, y\rangle \in I$, then we get

$$
f(\langle\Phi(A) y, y\rangle) \geq f(s)+(\langle\Phi(A) y, y\rangle-s) f^{\prime}(s)
$$

for any $s \in I$ that can be written as

$$
(s-\langle\Phi(A) y, y\rangle) f^{\prime}(s) \geq f(s)-f(\langle\Phi(A) y, y\rangle)
$$


for any $s \in I$.

Let $y \in K,\|y\|=1$. Using the continuous functional calculus for the operator $A$ we have

$$
A f^{\prime}(A)-\langle\Phi(A) y, y\rangle f^{\prime}(A) \geq f(A)-f(\langle\Phi(A) y, y\rangle) 1_{H} .
$$

Since $\Phi \in P_{N}[\mathcal{B}(H), \mathcal{B}(K)]$, then by taking the functional $\Phi$ in the inequality (3.3) we have

$$
\begin{gathered}
\Phi\left(A f^{\prime}(A)\right)-\langle\Phi(A) y, y\rangle \Phi\left(f^{\prime}(A)\right) \\
\quad \geq \Phi(f(A))-f(\langle\Phi(A) y, y\rangle) 1_{K}
\end{gathered}
$$

for any $y \in K,\|y\|=1$.

This is an inequality of interest in itself.

Taking the inner product in (3.4) we obtain the desired result (3.1).

Corollary 2. Let $I$ be an interval and $f: I \rightarrow \mathbf{R}$ be a convex and differentiable function on $I$ whose derivative $f^{\prime}$ is continuous on $I$. If $\Psi \in$ $P[\mathcal{B}(H), \mathcal{B}(K)]$ with $\Psi\left(1_{H}\right) \in \mathcal{B}^{++}(K)$ and $A$ is a selfadjoint operators on the Hilbert space $H$ with $\operatorname{Sp}(A) \subset I$, then

$$
\begin{aligned}
0 & \leq \frac{\langle\Psi(f(A)) v, v\rangle}{\left\langle\Psi\left(1_{H}\right) v, v\right\rangle}-f\left(\frac{\langle\Psi(A) v, v\rangle}{\left\langle\Psi\left(1_{H}\right) v, v\right\rangle}\right) \\
& \leq \frac{\left\langle\Psi\left(A f^{\prime}(A)\right) v, v\right\rangle}{\left\langle\Psi\left(1_{H}\right) v, v\right\rangle}-\frac{\langle\Psi(A) v, v\rangle}{\left\langle\Psi\left(1_{H}\right) v, v\right\rangle} \frac{\left\langle\Psi\left(f^{\prime}(A)\right) v, v\right\rangle}{\left\langle\Psi\left(1_{H}\right) v, v\right\rangle}
\end{aligned}
$$

for any $v \in K$ with $v \neq 0$.

The proof follows from the inequality (3.1) by a similar argument to the one from the proof of Corollary 1 and the details are omitted.

Let $\Phi: \mathcal{B}(H) \rightarrow \mathcal{B}(K)$ be a normalised positive linear map.

(i) If $A$ is a selfadjoint operator on $H$, then we have

$$
\begin{aligned}
0 & \leq\langle\Phi(\exp (A)) y, y\rangle-\exp (\langle\Phi(A) y, y\rangle) \\
& \leq\langle\Phi(A \exp (A)) y, y\rangle-\langle\Phi(A) y, y\rangle\langle\Phi(\exp (A)) y, y\rangle
\end{aligned}
$$

for all $y \in K,\|y\|=1$.

(ii) If $A$ is a positive (positive definite) operator on a Hilbert space $H$, then for any $p \geq 1(p \in(-\infty, 0))$ we have

$$
\begin{aligned}
0 & \leq\left\langle\Phi\left(A^{p}\right) y, y\right\rangle-\langle\Phi(A) y, y\rangle^{p} \\
& \leq p\left[\left\langle\Phi\left(A^{p}\right) y, y\right\rangle-\langle\Phi(A) y, y\rangle\left\langle\Phi\left(A^{p-1}\right) y, y\right\rangle\right]
\end{aligned}
$$


for all $y \in K,\|y\|=1$.

If $A$ is a positive operator on a Hilbert space $H$, then for any $p \in(0,1)$ we have

$$
\begin{aligned}
0 & \leq\langle\Phi(A) y, y\rangle^{p}-\left\langle\Phi\left(A^{p}\right) y, y\right\rangle \\
& \leq p\left[\langle\Phi(A) y, y\rangle\left\langle\Phi\left(A^{p-1}\right) y, y\right\rangle-\left\langle\Phi\left(A^{p}\right) y, y\right\rangle\right]
\end{aligned}
$$

for all $y \in K,\|y\|=1$.

(iii) If $A$ is a positive definite operator on a Hilbert space $H$, then

$$
\begin{aligned}
0 & \leq \ln (\langle\Phi(A) y, y\rangle)-\langle\Phi(\ln A) y, y\rangle \\
& \leq\langle\Phi(A) y, y\rangle\left\langle\Phi\left(A^{-1}\right) y, y\right\rangle-1
\end{aligned}
$$

for all $y \in K,\|y\|=1$.

Let $P_{j} \in \mathcal{B}(H), j=1, \ldots, k$ be contractions with the property (2.14). If $f: I \rightarrow \mathbf{R}$ is a convex function on the interval $I$ and $A$ is selfadjoint operator whose spectrum $\operatorname{Sp}(A)$ is contained in $I$, then we have by (3.1) that

$$
\begin{aligned}
0 & \leq\left\langle\sum_{j=1}^{k} P_{j}^{*} f(A) P_{j} y, y\right\rangle-f\left(\sum_{j=1}^{k}\left\langle P_{j}^{*} A P_{j} y, y\right\rangle\right) \\
& \leq\left\langle\sum_{j=1}^{k} P_{j}^{*} A f^{\prime}(A) P_{j} y, y\right\rangle-\left\langle\sum_{j=1}^{k} P_{j}^{*} A P_{j} y, y\right\rangle\left\langle\sum_{j=1}^{k} P_{j}^{*} f^{\prime}(A) P_{j} y, y\right\rangle
\end{aligned}
$$

for all $y \in K,\|y\|=1$. This is a generalization of (1.2).

In particular, if $A$ is a selfadjoint operator on $H$, then we have

$$
\begin{aligned}
0 & \leq\left\langle\sum_{j=1}^{k} P_{j}^{*} \exp (A) P_{j} y, y\right\rangle-\exp \left(\sum_{j=1}^{k}\left\langle P_{j}^{*} A P_{j} y, y\right\rangle\right) \\
& \leq\left\langle\sum_{j=1}^{k} P_{j}^{*} A \exp (A) P_{j} y, y\right\rangle-\left\langle\sum_{j=1}^{k} P_{j}^{*} A P_{j} y, y\right\rangle\left\langle\sum_{j=1}^{k} P_{j}^{*} \exp P_{j} y, y\right\rangle
\end{aligned}
$$

for all $y \in K,\|y\|=1$.

If $A$ is a positive (positive definite) operator on a Hilbert space $H$, then for any $p \geq 1(p \in(-\infty, 0))$ we have

$$
\begin{aligned}
0 & \leq\left\langle\sum_{j=1}^{k} P_{j}^{*} A^{p} P_{j} y, y\right\rangle-\left(\sum_{j=1}^{k}\left\langle P_{j}^{*} A P_{j} y, y\right\rangle\right)^{p} \\
& \leq p\left[\left\langle\sum_{j=1}^{k} P_{j}^{*} A^{p} P_{j} y, y\right\rangle-\left\langle\sum_{j=1}^{k} P_{j}^{*} A P_{j} y, y\right\rangle\left\langle\sum_{j=1}^{k} P_{j}^{*} A^{p-1} P_{j} y, y\right\rangle\right]
\end{aligned}
$$


for all $y \in K,\|y\|=1$. However, when $p \in(0,1)$ and $A$ is a positive, then

$$
\begin{aligned}
0 & \leq\left(\sum_{j=1}^{k}\left\langle P_{j}^{*} A P_{j} y, y\right\rangle\right)^{p}-\left\langle\sum_{j=1}^{k} P_{j}^{*} A^{p} P_{j} y, y\right\rangle \\
& \leq p\left[\left\langle\sum_{j=1}^{k} P_{j}^{*} A P_{j} y, y\right\rangle\left\langle\sum_{j=1}^{k} P_{j}^{*} A^{p-1} P_{j} y, y\right\rangle-\left\langle\sum_{j=1}^{k} P_{j}^{*} A^{p} P_{j} y, y\right\rangle\right],
\end{aligned}
$$

for all $y \in K,\|y\|=1$.

If $A$ is a positive definite operator on $H$, then

$$
\begin{aligned}
0 & \leq \ln \left(\sum_{j=1}^{k}\left\langle P_{j}^{*} A P_{j} y, y\right\rangle\right)-\left\langle\sum_{j=1}^{k} P_{j}^{*}(\ln A) P_{j} y, y\right\rangle \\
& \leq\left\langle\sum_{j=1}^{k} P_{j}^{*} A P_{j} y, y\right\rangle\left\langle\sum_{j=1}^{k} P_{j}^{*} A^{-1} P_{j} y, y\right\rangle-1
\end{aligned}
$$

for all $y \in K,\|y\|=1$.

These inequalities generalize the corresponding results from (1.4)-(1.6).

\section{References}

[1] M. D. Choi, Positive linear maps on C*-algebras. Canad. J. Math. 24, pp. 520-529, (1972).

[2] S. S. Dragomir, Some reverses of the Jensen inequality for functions of selfadjoint operators in Hilbert spaces. J. Inequal. Appl., Art. ID $496821,15,(2010)$.

[3] S. S. Dragomir, Operator Inequalities of the Jensen, Čebyšev and Grüss Type. Springer Briefs in Mathematics. Springer, New York, xii+121 pp. ISBN: 978-1-4614-1520-6, (2012).

[4] S. S. Dragomir and N. M. Ionescu, Some converse of Jensen's inequality and applications. Rev. Anal. Numér. Théor. Approx. 23, No. 1, pp. 7178. MR:1325895 (96c:26012), (1994).

[5] C. A. McCarthy, $c_{p}$, Israel J. Math., 5, pp. 249-271, (1967).

[6] B. Mond and J. Pečarić, Convex inequalities in Hilbert space, Houston J. Math., 19, pp. 405-420, (1993). 
[7] J. Pečarić, T. Furuta, J. Mićić Hot and Y. Seo, Mond-Pečarić Method in Operator Inequalities. Inequalities for Bounded Selfadjoint Operators on a Hilbert Space, Element, Zagreb, (2005).

\section{S. S. Dragomir}

Mathematics,

College of Engineering \& Science

Victoria University, P. O. Box 14428

Melbourne City,

MC 8001,

Australia

e-mail : sever.dragomir@vu.edu.au 